\title{
Frequency, risk factors, and outcomes in patients with significant carotid artery disease admitted to King Abdulaziz Medical City, Riyadh with Ischemic Stroke
}

Misealreem A. Shaheen, MBBS, Areej A. Albelali, MBBS, Raghad M. AlKanhal, MBBS, Muneera K. AlSaqabi, MBBS, Raghad M. AlTurki, MBBS, Raghad S. AlAskar, MBBS, Altaf H. Khan, MS, Ismail A. Khatri, MD, FAAN.

\begin{abstract}

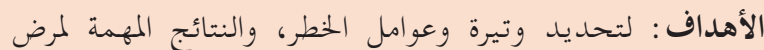

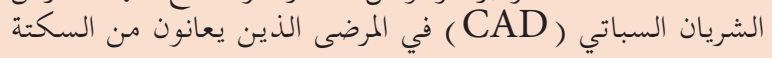
الدماغية.

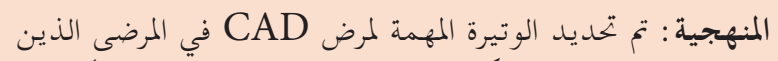

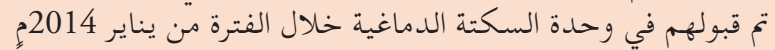

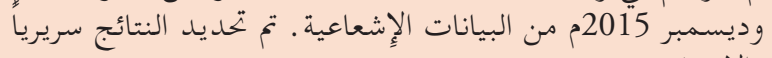
والإِشعاعي.

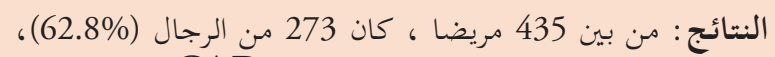

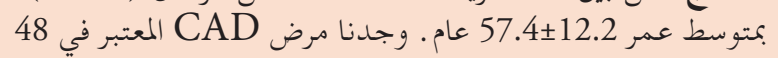

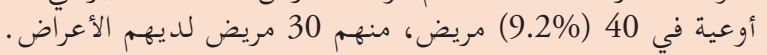

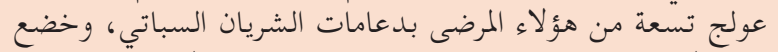

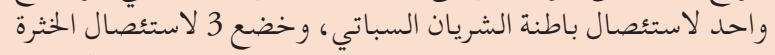

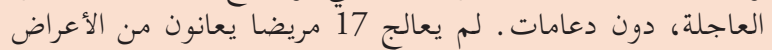

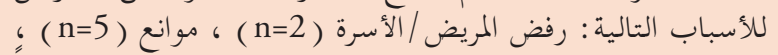

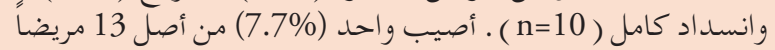

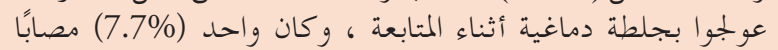

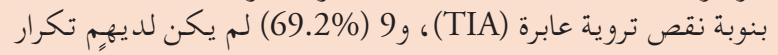

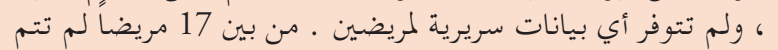

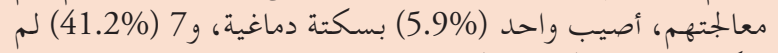
يتكرر ، وفقدو 9 (52.9) للمتابعة .

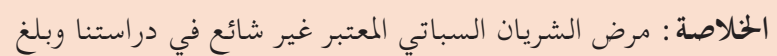

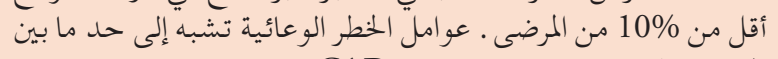

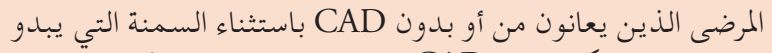

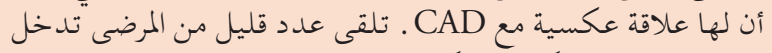

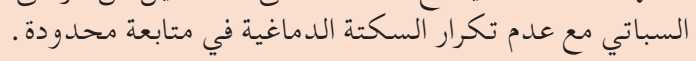

Objectives: To determine the frequency, risk factors, and outcomes of significant carotid artery disease $(\mathrm{CAD})$ in patients with ischemic stroke.

Methods: The frequency of significant CAD in patients admitted to the Stroke Unit between January 2014 and December 2015 was determined from radiological data. Outcomes were determined clinically and radiologically.

Results: Among 435 patients, 273 were men (62.8\%), with a mean age of $57.4 \pm 12.2$ years. Significant CAD was found in 48 vessels in $40(9.2 \%)$ patients, of which 30 patients were symptomatic. Nine of these patients were treated with carotid artery stenting, one underwent carotid endarterectomy, and 3 underwent an urgent thrombectomy, without stenting. Seventeen symptomatic patients were not treated for the following reasons: patient/family refusal $(n=2)$, contraindications $(\mathrm{n}=5)$, and complete occlusion $(n=10)$. One $(7.7 \%)$ of the 13 treated patients had an ipsilateral stroke on follow up, one $(7.7 \%)$ had contralateral transient ischemic attack (TIA), 9 $(69.2 \%)$ had no recurrence, and no clinical data were available for 2 patients. Among the 17 untreated patients, one $(5.9 \%)$ had an ipsilateral stroke, 7 (41.2\%) had no recurrence, and $9(52.9 \%)$ were lost to follow up.

Conclusions: Significant carotid artery disease is uncommon in our cohort found in less than $10 \%$ of patients. Vascular risk factors are more or less similar between patients with or without CAD except obesity which appears to have inverse relation with CAD. A small number of patients received carotid intervention with no recurrence of stroke at limited follow up.

Neurosciences 2019; Vol. 24 (4): 264-268 doi: 10.17712/nsj.2018.4.20190046

From King Abdulaziz Medical City, MNGHA (Khatri), King Saud bin Abdulaziz University for Health Sciences (Shaheen, Albelali, AlKanhal, AlSaqabi, AlTurki, AlAskar, Khatri), King Abdullah International Medical Research Center (Khan, Khatri), Riyadh, Kingdom of Saudi Arabia.

Received 26th May 2019. Accepted 24th July 2019.

Address correspondence and reprint request to: Dr. Ismail A. Khatri, Division of Neurology, Department of Medicine, King Abdulaziz Medical City, Ministry of National Guard Health Affairs, Riyadh, Kingdom of Saudi Arabia. Email: ismailkhatri@yahoo.com ORCID ID: https://orcid.org/0000-0002-0218-1840 
Strokes are the second leading cause of death Sworldwide. ${ }^{1}$ According to data from the Institute of Neurological and Communicative Disorders and Stroke, $22 \%$ of strokes can be attributed to carotid artery disease (CAD). ${ }^{2}$ Carotid artery disease is classified as extracranial or intracranial. There appear to be racial variations in the distribution of the 2 types of CAD, with a relatively higher incidence of intracranial disease in black and Hispanic individuals ${ }^{3}$ and a relatively higher incidence of extracranial atherosclerotic disease among Caucasians. ${ }^{4}$ Extracranial atherosclerosis has long been identified as a common source of emboli that can travel to the brain and cause a stroke. ${ }^{5}$ Intracranial atherosclerotic disease accounts for $30-50 \%$ of strokes in Asia. ${ }^{6}$ Carotid artery disease is classified into symptomatic and asymptomatic disease. Symptomatic patients with carotid stenosis experience a focal neurological deficit ipsilateral to stenosis. ${ }^{7}$ Asymptomatic carotid stenosis is not a life-threatening condition, with a mortality rate of $0.05 \%$ and a stroke recurrence rate of $1-2 \%$ or less annually. ${ }^{8}$ However, it is significantly associated with the development of lifethreatening cardiovascular events, as it co-exists with coronary artery disease in $40 \%$ of cases. ${ }^{8}$ Symptomatic $\mathrm{CAD}$ can be managed by medications alone, or medications with carotid artery stenting or carotid endarterectomy, depending on the individual patient. ${ }^{7}$ In acute cases, carotid thrombectomy without carotid artery stenting can be performed as part of endovascular treatment of an acute stroke, although this has not been well studied in randomized trials. A study by $\mathrm{Al}$ Rajeh et $\mathrm{al}^{9}$ on 500 Saudi stroke patients found that ischemic strokes accounted for $76.2 \%$ of the stroke cases. Determining the degree of carotid stenosis and type of CAD (i.e., symptomatic or asymptomatic) is crucial for patients' management and prognosis. ${ }^{7}$ Data on significant CAD among stroke patients in the Saudi population are lacking. The scarcity of data is cause for concern. In this study, we determined the frequency, risk factors, management and outcomes of significant CAD in ischemic stroke patients. We also determined the prevalence of symptomatic or asymptomatic disease.

Methods. This study was conducted in the stroke unit of King Abdulaziz Medical City, Ministry of National Guard Health Affairs, Riyadh, Kingdom

Disclosure. Authors have no conflict of interests, and the work was not supported or funded by any drug company. of Saudi Arabia. Patients admitted to the stroke unit between January 2014 and December 2015 were included. Significant CAD was defined as more than $50 \%$ stenosis confirmed on vascular imaging. If the patient had suffered an ischemic stroke or transient ischemic attack (TIA) and the ipsilateral vessel showed significant stenosis $(>50 \%)$, CAD was considered symptomatic.

The inclusion criteria included all ischemic stroke patients aged 18 years and older of both genders. Patients admitted to the neurology department were included in the study. Stroke patients admitted to any other department in the hospital were excluded. Additional exclusion criteria included patients with intracerebral hemorrhages, a final diagnosis other than a stroke, and missing important data.

Demographic, clinical, and radiological data were collected using a standard prespecified form. The descriptive statistics for the demographic and clinical characteristics are presented as the mean (median), with standard deviations for continuous variables and counts and percentages for categorical variables.

The study was approved by the Institutional Review Board of King Abdullah International Medical Research Center (KAIMRC) and King Saud bin Abdulaziz University for Health Sciences (KSAU-HS), Riyadh, Kingdom of Saudi Arabia. Due to retrospective, chart review nature of the study, the IRB waived the need for informed consent from the included patients.

Statistical analysis. Pearson's chi-square test or Fisher's exact test was used to detect clinical associations between the categorical variables and the outcome variable. To assess the association of demographics and clinical variables with the outcome variable, a general linear model, generalized estimation equation, and mixed methods were utilized as the study cohort was longitudinal. The significance level $\alpha$ was considered 0.05 if the probabilistic $p$-value was less than the assumed significance level. The statistical software packages SAS version 9.4 (SAS Institute Inc. Cary, NC, USA) or R version 3.4 (The R Project for Statistical Computing: free software) was used for data analysis. The data were analyzed using SPSS Statistics (SPSS Inc., Chicago, IL, USA) version 22.0. Fisher's exact test, the $\chi^{2}$ test, an independent sample t-test, Mann-Whitney test, one-way analysis of variance, and multiple logistic regression analysis were conducted.

Results. Of 726 stroke patients included in the study, 435 patients were included in the final analysis. The other patients were excluded for the following reasons: a hemorrhagic stroke ( $n=57$ patients), final 
Table 1 - Frequency of various risk factors in the whole group and significant carotid artery disease (CAD) and nonsignificant CAD subgroups.

\begin{tabular}{|c|c|c|c|}
\hline \multicolumn{4}{|c|}{ Carotid artery Group } \\
\hline \multirow[t]{3}{*}{ Risk factors } & $\begin{array}{l}\text { Nonsignificant } \\
\text { group }\end{array}$ & $\begin{array}{l}\text { Significant } \\
\text { group }\end{array}$ & $P$-value \\
\hline & $\mathrm{n}=395$ & $n=40$ & \\
\hline & \multicolumn{2}{|c|}{ n (\%) } & \\
\hline \multicolumn{4}{|l|}{ Gender } \\
\hline Male & $248(62.78)$ & $25(62.5)$ & 0.972 \\
\hline Female & $147(37.22)$ & $15(37.5)$ & \\
\hline \multicolumn{4}{|l|}{ Diabetes } \\
\hline Yes & $245(62.03)$ & $31(77.5)$ & 0.053 \\
\hline No & $150(37.97)$ & $9(22.5)$ & \\
\hline \multicolumn{4}{|l|}{ Obesity } \\
\hline Yes & $162(41.01)$ & $9(22.5)$ & 0.022 \\
\hline No & 233(58.99) & $31(77.5)$ & \\
\hline \multicolumn{4}{|l|}{ Smoking } \\
\hline Yes & $72(18.22)$ & $10(25.0)$ & 0.298 \\
\hline No & $323(81.78)$ & $30(75.0)$ & \\
\hline \multicolumn{4}{|l|}{ Prior stroke } \\
\hline Yes & $65(16.46)$ & $8(20)$ & 0.298 \\
\hline No & $330(83.54)$ & $32(80)$ & \\
\hline \multicolumn{4}{|l|}{ Dyslipidemia } \\
\hline Yes & $149(37.72)$ & $18(45)$ & 0.367 \\
\hline No & $246(62.28)$ & $22(55)$ & \\
\hline \multicolumn{4}{|l|}{ Migraine } \\
\hline Yes & $5(1.27)$ & $1(2.5)$ & 0.4414 \\
\hline No & $390(98.73)$ & $39(97.5)$ & \\
\hline \multicolumn{4}{|l|}{ Hypertension } \\
\hline Yes & $282(71.39)$ & $33(82.5)$ & 0.134 \\
\hline No & $113(28.61)$ & $7(17.5)$ & \\
\hline \multicolumn{4}{|c|}{ Ischemic heart disease } \\
\hline Yes & $56(14.18)$ & $8(20)$ & 0.322 \\
\hline No & $339(85.82)$ & $32(80)$ & \\
\hline \multicolumn{4}{|c|}{ Transient ischemic attack } \\
\hline Yes & $10(2.53)$ & $2(5)$ & 0.304 \\
\hline No & 385 (97.47) & $38(95)$ & \\
\hline
\end{tabular}

diagnosis other than a stroke ( $\mathrm{n}=85$ patients), missing data on carotid artery disease $(n=30)$, and incomplete medical records $(\mathrm{n}=119)$.

There were 273 men (62.8\%) and 162 women (37.2\%), with a mean age of $57.4 \pm 12.2$ years. In terms of stroke-related risk factors, 315 (72.4\%) patients had hypertension, $276(63.4 \%)$ patients had diabetes, and $171(39.3 \%)$ patients were obese (Table 1). A comparison of risk factors between patients with significant and nonsignificant CAD is shown in Table 2, with the estimated parameters, standard errors, $p$-values, and odds ratios, with their $95 \%$ confidence intervals. In the univariate analysis, obesity showed an inverse relationship with significant CAD $(p=0.022)$ (Table 1). This finding persisted in the logistic regression analysis, with obesity showing an inverse relation with significant CAD ( $p=0.0087)$. There were no betweengroup differences in any of the other risk factors (Table 2, Figure 1). Obesity as a risk factor for significant CAD was associated with lower odds (36\%) as compared to nonobese individuals. Significant $\mathrm{CAD}$ was found in 48 vessels in $40(9.2 \%)$ patients, of which $30(6.9 \%)$ patients were symptomatic. Of these 30 symptomatic patients, $9(30 \%)$ were treated with carotid artery stenting, one $(3.33 \%)$ underwent endarterectomy, and $3(10 \%)$ underwent an urgent thrombectomy, without stenting. The other 17 (56\%) symptomatic patients were not treated for the following reasons: patient/ family refusal $(n=2,11.8 \%)$, contraindications to the intervention $(\mathrm{n}=5,29.4 \%)$, and complete occlusion $(n=10,58.8 \%)$. These results are summarized in Figure 2.

As shown by the clinical and radiological follow up of the treated $(n=13)$ and untreated $(n=17)$ patients, one $(7.7 \%)$ patient had an ipsilateral stroke on follow up, and one $(7.7 \%)$ patient had a contralateral TIA at the 6-months follow up. Nine (69.2\%) patients had no recurrence, and no clinical data were available on $2(16.9 \%)$ patients. Among the untreated 17 vessels, one $(5.9 \%)$ patient had an ipsilateral stroke, 7 (41.2\%) patients had no recurrence, and $9(52.9 \%)$ patients were lost to follow up.

Discussion. In the West, CAD is a strong risk factor for a stroke, accounting for approximately 20-22\% of stroke cases. ${ }^{10} \mathrm{~A}$ recent study in Pakistan showed that CAD was present in $56 \%$ of stroke patients, making it a significant risk factor in this population. ${ }^{11}$ In contrast to the literature, the frequency of significant CAD was only $9 \%$ in our cohort, of which $6.9 \%$ of patients were symptomatic. A study in Taiwan reported a similar low frequency, where ischemic strokes resulting from $\mathrm{CAD}$ were found in only $6 \%$ of the cohort. ${ }^{12}$ A recent Saudi study that compared diabetic and nondiabetic patients detected significant carotid artery stenosis in 13.4\% of all patients. ${ }^{13}$ A study in Iran found that most large vessel strokes $(69 \%)$ were attributable to extracranial CAD. ${ }^{14}$ These wide variations in the frequency of significant $\mathrm{CAD}$ are difficult to explain and warrant large multicenter, multinational studies to ascertain the reasons for the disparity in different populations.

Among the symptomatic patients in our cohort, less than $50 \%$ underwent a carotid intervention, with the other patients either having contraindications or the patient or family refusing an intervention. We also found that a large number of symptomatic vessels were completely occluded, which may suggest long-standing 
Table 2 - A comparison of risk factors in patients with significant and nonsignificant carotid artery disease (CAD).

\begin{tabular}{lccccccc}
\hline Parameter & & \multicolumn{6}{c}{ Analysis of Penalized Maximum Likelihood Estimates } \\
& & DF & Estimate & Standard Error & Wald chi-square & Pr > chi-square & $\begin{array}{c}\text { Odds ratio } \\
\text { (95\% confidence intervals) }\end{array}$ \\
\hline Gender & Female vs. Male & 1 & 0.5363 & 0.3941 & 1.8518 & 0.1736 & $1.71(0.76-3.77)$ \\
Diabetes & Yes vs. No & 1 & 0.5828 & 0.4132 & 1.9898 & 0.1584 & $1.79(0.81-4.28)$ \\
Obesity & Yes vs. No & 1 & -1.0310 & 0.3931 & 6.8782 & 0.0087 & $0.36(0.15-0.76)$ \\
Smoking & Yes vs. No & 1 & 0.6932 & 0.4250 & 2.6612 & 0.1028 & $2.00(0.84-4.58)$ \\
Prior stroke & Yes vs. No & 1 & 0.3198 & 0.4374 & 0.5346 & 0.4647 & $1.38((0.55-3.16)$ \\
Dyslipidemia & Yes vs. No & 1 & 0.3369 & 0.3418 & 0.9713 & 0.3244 & $1.40(0.71-2.76)$ \\
Migraine & Yes vs. No & 1 & 0.9752 & 1.0577 & 0.8501 & 0.3565 & $2.65(0.25-15.33)$ \\
Hypertension & Yes vs. No & 1 & 0.4218 & 0.4593 & 0.8434 & 0.3584 & $1.53(0.63-4.05)$ \\
Ischemic heart disease & Yes vs. No. & 1 & 0.2843 & 0.4339 & 0.4295 & 0.5122 & $1.33(0.54-3.01)$ \\
Transient ischemic attack & Yes vs. No & 1 & 0.6300 & 0.7940 & 0.6297 & 0.4275 & $1.88(0.33-7.43)$ \\
Atrial fibrillation & Yes vs. No & 1 & 0.8909 & 0.6420 & 1.9259 & 0.1652 & $2.44(0.60-7.67)$ \\
\hline
\end{tabular}

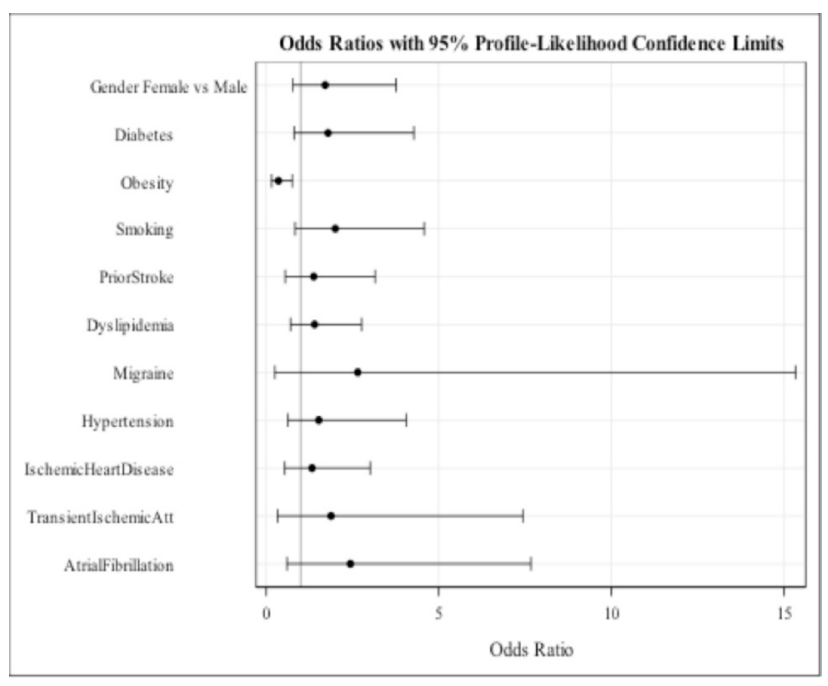

Figure 1 - Likelihood ratios of risk factors in patients with significant and nonsignificant carotid artery disease $(\mathrm{CAD})$.

disease that became symptomatic with an incident stroke. When this study was performed, there was equipoise for carotid artery stenting and endarterectomy with no clear preference of one procedure over the other. Most of our treated patients had carotid artery stenting as compared to carotid endarterectomy. This likely point to institutional bias toward carotid artery stenting. Our follow-up data were also not complete, as many patients were lost to follow up or were not followed for a long duration. The maximum follow-up time was 1 year. Among the patients treated for carotid disease, one had an ipsilateral stroke, and one had a contralateral TIA. Among the untreated group, one patient had a contralateral stroke. At the last follow up, $69 \%$ of patients in the treated group had no recurrence as compared with $41 \%$ in the untreated group.

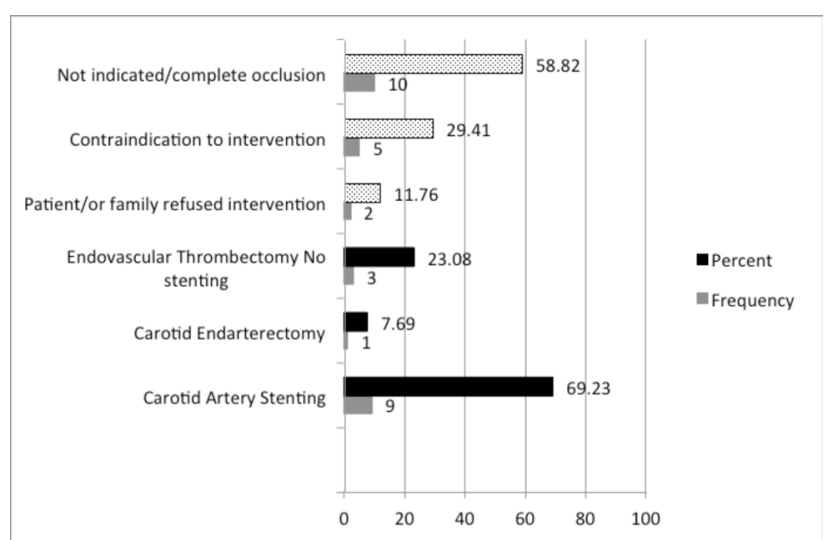

Figure 2 - Pattern of intervention in the significant group, treated group (dark black bars), and nontreated group (dotted bars).

However, $17 \%$ in the treated group and $53 \%$ patients in the untreated group were lost to follow up.

We found a significant increase in the frequency of vascular risk factors in our cohort as compared with that reported in 2 previous studies conducted in Saudi Arabia in the 1990s. ${ }^{9}, 15$ These studies reported hypertension in $41-56 \%$ of their cohorts as compared with $72.4 \%$ in our cohort. This finding may suggest an overall increase in the prevalence of hypertension or a higher number of untreated patients in the Saudi population. Zafar et $\mathrm{a}^{13}$ also found an increased frequency of vascular risk factors among stroke patients. A recent national survey in Saudi Arabia of more than 10,000 individuals older than 15 years found that approximately $55 \%$ of the population had hypertension or borderline hypertension. ${ }^{16}$ In our cohort, the prevalence of diabetes was $63.5 \%$, which points to a significant increase in comparison to the previous frequencies of $42 \%$ and $25 \%$ reported in Saudi populations. ${ }^{9,15}$ The incidence of ischemic heart disease 
has also increased $(14.7 \%)$ as compared with that found in the previous Saudi study (10\%). ${ }^{15}$ The median age of our patients was 57 years, which was somewhat younger than the mean ages of 61 and 63 years reported in the earlier studies. ${ }^{9,15} \mathrm{~A}$ marked increase in cerebrovascular risk factors may explain the younger age of the patients in our cohort.

Other than established cerebrovascular risk factors, various other factors, such as obesity and lifestyle changes, can play a role in stroke risk. In 1992, the obesity rate in Saudi Arabia was estimated to be $16.4 \%$, whereas it was $52.9 \%$ in $2016 .{ }^{17}$ In our study, $39.3 \%$ of the patients were obese. In general, obesity has a negative impact on cardiovascular morbidity. However, we found an interesting paradox, with obesity appearing to play a protective role against significant CAD in our cohort. Obesity was inversely related to significant carotid artery stenosis in stroke patients, reducing the risk of CAD up to $36 \%(p=0.0087)$. A previous study also reported that obesity was associated with reduced mortality in stroke patients and with a reduced risk of readmission for recurrent strokes. ${ }^{18}$

Ourstudy hasseverallimitations. It was a retrospective cohort study, and most data were extracted from the hospital files. Some CAD patients were excluded due to missing files and others were excluded due to missing or incomplete data. The documentation for all stroke patients was also not standardized. In addition, our cohort was from a single tertiary care center, which caters mostly to members of the Saudi National Guard, although emergency care is provided to all patients, regardless of their affiliation with the National Guard. Thus, our data are likely not generalizable to the entire Saudi population. Among the patients diagnosed with CAD, many patients were lost to follow up, and longitudinal data on outcomes were not available.

In conclusions, carotid artery disease was an uncommon cause of ischemic stroke in our population with less than $10 \%$ of ischemic stroke patients having significant CAD. Although traditional vascular risk factors did not differ among patients with or without CAD, obesity showed an interesting and as yet unexplained paradoxical role with $\mathrm{CAD}$, providing possible protection against carotid disease among stroke patients. A small number of patients with symptomatic $\mathrm{CAD}$ underwent intervention with no recurrence of stroke at last follow up.

We recommend multicenter studies in Saudi Arabia to determine the frequency of significant CAD, as well as the reasons for the apparent discrepancy in the frequency as compared with that in other regions of the world. There is an urgent need for action to address vascular risk factors in the Saudi population to decrease the risk of strokes and stroke-related morbidity and mortality.

\section{References}

1. World Health Organization. The top 10 causes of death [Internet]. Geneva (CH): World Health Organization; 2016. [cited 5 April 2016]. Available from: http://www.who.int/ mediacentre/factsheets/fs310/en/.

2. Zhu C, Norris J. Role of carotid stenosis in ischemic stroke. Stroke 1990; 21: 1131-1134.

3. Rincon F, Sacco RL, Kranwinkel G, Xu Q, Paik MC, BodenAlbala B, et al. Incidence and risk factors of intracranial atherosclerotic stroke: The Northern Manhattan Stroke Study. Cerebrovascular Dis 2009; 28: 65-71.

4. Rockman C, Maldonado T, Jacobowitz G, Berger J, Adelman M, Riles T. The prevalence of carotid artery stenosis varies significantly by Race. J Vasc Surg 2013; 57: 327-337.

5. Eliasziw M. Early risk of stroke after a transient ischemic attack in patients with internal carotid artery disease. CMAJ 2004; 170: 1105-1109.

6. Turan TN, LeMatty T, Martin R, Chimowitz MI, Rumboldt Z, Spampinato MV, et al. Characterization of intracranial atherosclerotic stenosis using high-resolution MRI study-rationale and design. Brain Behav 2015; 5: e00397.

7. Lanzino G, Rabinstein A, Brown R. Treatment of carotid artery stenosis: medical therapy, surgery, or stenting? Mayo Clin Proc 2009; 84: 362-368.

8. Chatzikonstantinou A, Wolf M, Schaefer A, Hennerici M. Asymptomatic and symptomatic carotid stenosis: an obsolete classification? Stroke Res Treat 2012; 2012: 340798.

9. al Rajeh S, Awada A, Niazi G, Larbi E. Stroke in a Saudi Arabian National Guard community. Analysis of 500 consecutive cases from a population-based hospital. Stroke 1993; 24: 1635-1639.

10. Sacco RL, Ellenberg JH, Mohr JP, Tatemichi TK, Hier DB, Price TR, et al. Infarcts of undetermined cause: the NINCDS Stroke Data Bank. Ann Neurol 1989; 25: 382-390.

11. Afridi A, Afridi Z, Afridi F, Afridi A. Frequency of carotid artery stenosis in ischemic stroke patients. J Med Sci 2017; 25: 340-343.

12. Tan T, Chang K, Liou C, Schminke U. Prevalence of carotid artery stenosis in Taiwanese patients with one ischemic stroke. $J$ Clin Ultrasound 2005; 33: 1-4.

13. Zafar A, Al-Khamis FA, Al-Bakr AI, Alsulaiman AA, Msmar AH. Risk factors and subtypes of acute ischemic stroke. A study at King Fahd Hospital of the University. Neurosciences (Riyadh) 2016; 21: 246-251.

14. Saber H, Amiri A, Thrift AG, Stranges S, Bavarsad Shahripour R, Farzadfard MT, et al. Epidemiology of Intracranial and Extracranial Large Artery Stenosis in a Population-Based Study of Stroke in the Middle East. Neuroepidemiology 2017; 48: 188-192.

15. Yaqub BA, Shamena AR, KolawoleTM, Patel PJ. Cerebrovascular disease in Saudi Arabia. Stroke 1991; 22: 1173-1176.

16. El Bcheraoui C, Memish ZA, Tuffaha M, Daoud F, Robinson $\mathrm{M}$, Jaber $\mathrm{S}$, et al. Hypertension and its associated risk factors in the kingdom of saudi arabia, 2013: a national survey. Int J Hypertens 2014; 2014: 564679.

17. Alqarni SS. A review of prevalence of obesity in Saudi Arabia. $J$ Obes Eat Disord 2016; 2: 25.

18. Andersen KK, Olsen TS. The obesity paradox in stroke: lower mortality and lower risk of readmission for recurrent stroke in obese stroke patients. Int J Stroke 2015; 10: 99-104. 